\title{
Quadriceps muscle deoxygenation during functional electrical stimulation in adults with spinal cord injury
}

\author{
Y Bhambhani*,1,3, C Tuchak ${ }^{2}$, R Burnham ${ }^{2}$, J Jeon $^{3}$ and R Maikala ${ }^{1}$ \\ ${ }^{1}$ Faculty of Rehabilitation Medicine, University of Alberta, Edmonton, Canada, T6G 2G4; ${ }^{2}$ Faculty of Medicine, \\ University of Alberta, Edmonton, Canada, T6G 2G4; ${ }^{3}$ Faculty of Physical Education and Recreation, University of \\ Alberta, Edmonton, Canada, T6G $2 G 4$
}

\begin{abstract}
Study design: Cross-sectional study comparing healthy subjects with age and gender matched subjects with spinal cord injury (SCI, injury levels from C5 to T12).

Objectives: To compare the acute cardiorespiratory responses and muscle oxygenation trends during functional electrical stimulation (FES) cycle exercise and recovery in the SCI and healthy subjects exercising on a mechanical cycle ergometer.

Setting: Seven volunteers in each group participated in one exercise test at the Rick Hansen Center, University of Alberta, Edmonton, Canada.

Methods: Both groups completed a stagewise incremental test to voluntary fatigue followed by 2 min each of active and passive recovery. Cardiorespiratory responses were continuously monitored using an automated metabolic cart and a wireless heart rate monitor. Tissue absorbency, an index of muscle oxygenation, was monitored non-invasively from the vastus lateralis using near infrared spectroscopy.

Results: The healthy subjects showed significant $(P<0.05)$ increases in the oxygen uptake $\left(\dot{\mathrm{VO}}_{2}\right)$, heart rate $(\mathrm{HR})$ and ventilation rate $\left(\dot{\mathrm{V}}_{\mathrm{E}}\right)$ from rest to maximal exercise. The SCI subjects showed a twofold increase in $\dot{\mathrm{V}}_{2}(P>0.05)$, a threefold increase in $\dot{\mathrm{V}}_{\mathrm{E}}(P<0.05)$ and a 5 beats/min increase in HR $(P>0.05)$ from the resting value. The SCI subjects demonstrated a lesser degree $(P<0.05)$ of muscle deoxygenation than the healthy subjects during the transition from rest to exercise. Regression analysis indicated that the rate of decline in muscle deoxygenation with respect to the $\mathrm{VO}_{2}$ was significantly $(P<0.05)$ faster in the SCI subjects compared to healthy subjects.

Conclusions: FES exercise in SCI subjects elicits: (a) modest increases in the cardiorespiratory responses when compared to resting levels; (b) lower degree of muscle deoxygenation during maximal exercise, and (c) faster changes in muscle deoxygenation with respect to the $\dot{\mathrm{V}} \mathrm{O}_{2}$ during exercise when compared to healthy subjects.
\end{abstract}

Spinal Cord (2000) 38, 630-638

Keywords: quadriplegia; paraplegia; functional electrical stimulation; vastus lateralis, muscle metabolism

\section{Introduction}

Functional electrical stimulation (FES) is a technique which allows persons with spinal cord injury (SCI) to perform functional tasks such as rudimentary walking, ${ }^{1}$ rowing ${ }^{2}$ and cycle exercise ${ }^{3,4}$ with their paralyzed extremities. Studies that have evaluated the acute physiological responses to incremental FES cycle exercise in persons with SCI have indicated that the oxygen uptake $\left(\dot{\mathrm{V}}_{2}\right)$, ventilation rate $\left(\dot{\mathrm{V}}_{\mathrm{E}}\right)$,

*Correspondence: Dr Y Bhambhani, Faculty of Rehabilitation Medicine, University of Alberta, Edmonton, Canada, T6G 2G4 cardiac output ( $\dot{\mathrm{Q}})$, heart rate (HR) and stroke volume (SV) show modest increases from resting levels in both paraplegics and quadriplegics. ${ }^{3-6}$ However, the factors contributing to the increase in whole body $\dot{\mathrm{VO}}_{2}$ during FES cycle exercise in subjects with SCI are not known. It is possible that the elevated $\dot{\mathrm{VO}}_{2}$ during this exercise mode in SCI subjects could be the result of: (a) an increase in aerobic metabolism of the muscles that are electrically stimulated, and/or (b) an elevated metabolic rate in the tissues indirectly involved during such involuntary exercise; eg the heart and lungs. 
Early muscle fatigue is a common factor limiting the success of FES exercise programs. It is likely that both central and peripheral factors which determine the $\mathrm{VO}_{2}$ of the tissue are implicated in this phenomenon. Individuals with SCI have a hypokinetic circulation; ${ }^{5,6}$ ie a reduced $\dot{Q}$ for a given $\mathrm{VO}_{2}$. This results in a significant reduction in oxygen transported to the tissues when compared to their able-bodied counterparts. The hypokinetic circulation has been attributed to a significantly lower SV and HR, particularly in individuals with quadriplegia. ${ }^{5,6}$ It has been reported that following SCI, skeletal muscle undergoes significant physiologic and histologic changes. ${ }^{7-9}$ These include a reduced capillary density, a lower mitochondrial density in the existing motor units, and a relative increase in Type II motor units which have a lower oxidative capacity than the Type I motor units. Collectively, the central and peripheral changes resulting from SCI could reduce the muscle oxygenation levels thereby resulting in premature fatigue. It is important, therefore, that localized muscle oxygenation levels be evaluated during FES exercise in persons with SCI. This would also lead to a better understanding of the acute physiological effects of this exercise mode on paralyzed muscle, thereby enabling us to design appropriate rehabilitation programs to minimize fatigue and maximize the benefits to the client.

Near Infra-red Spectroscopy (NIRS) is a valid and reliable technique for measuring relative changes in skeletal muscle oxygenation during exercise. ${ }^{10,11}$ NIRS is based on the differential absorption properties of hemoglobin and myoglobin in the near infra-red range, ie at wave lengths between 700 and $1000 \mathrm{nM}$. At $760 \mathrm{~nm}$ hemoglobin is in the deoxygenated form, whereas at $850 \mathrm{nM}$ it is in the oxygenated state. The difference in NIRS signal between these two wave lengths reflects the relative change in oxygenation in the small blood vessels namely the arterioles, capillaries and venules. The sum signal at these two wave lengths indicates the relative change in blood volume. NIRS is unable to differentiate between the hemoglobin and myoglobin signals in vivo because the absorption spectra of these two chromophores (light absorbing compounds) overlap. However, in vitro studies have demonstrated that approximately $75 \%$ of the deoxygenation is due to the release of oxygen by hemoglobin, while the balance is due to the release of oxygen by myoglobin. ${ }^{12}$ One of the limitations of NIRS is that localized muscle oxygen uptake cannot be determined because the path length of the NIRS signal cannot be quantified during dynamic exercise. ${ }^{11}$ However, since NIRS is able to identify relative changes in skeletal muscle oxygenation during exercise, it has been used extensively to evaluate trends in skeletal muscle oxygenation during a variety of exercise modes in healthy subjects as well as a variety of clinical populations. ${ }^{13}$ This includes patients with heart failure, ${ }^{1,14-16}$ peripheral vascular disease ${ }^{17}$ and chronic compartmental syndrome. ${ }^{18,19}$
To date, NIRS has not been used to examine muscle oxygenation trends in persons with SCI. This study was designed to compare the acute cardiorespiratory, metabolic and muscle oxygenation responses between healthy subjects performing cycle ergometry with those of SCI subjects during FES cycle exercise. In light of the lower oxygen transport, delivery and utilization characteristics that have been well documented in SCI subjects, it was hypothesized that the degree and rate of deoxygenation in the vastus lateralis muscle measured by NIRS would be significantly lower in the SCI subjects compared to the healthy counterparts.

\section{Methods}

\section{Subjects}

Written informed consent was obtained from seven healthy and seven SCI volunteers for this study. The subjects in the two groups were matched for age and gender. Some pertinent physical characteristics are presented in Table 1. Each subject completed the Physical Activity-Readiness Questionnaire (PAR-Q) prior to testing to screen for medical/physical problems that could preclude exercise testing. ${ }^{20}$ Any individual who responded positively to the PAR-Q was not allowed to participate in the study. All the SCI subjects had an upper motor neuron injury with complete lower limb paralysis. At the time of testing, four of these subjects were training on the FES cycle ergometer, two had some experience on this instrument and one had never used it. The exclusion criteria for the SCI subjects were those that preclude FES: (a) autonomic dysreflexic response to FES; ${ }^{21}$ ie greater than $50 \%$ rise of mean arterial blood pressure above resting value or a blood pressure $>180 / 110$; (b) pressure sore on buttock or heel precluding $30 \mathrm{~min}$ of sustained sitting; (c) less than $90^{\circ}$ of hip flexion or knee flexion range of motion; (d) fecal or urinary incontinence with electrical stimulation; (e) severe leg spasticity (Modified Ashworth Scale grade 4); and (f) sensory sparing such that FES stimulation causes intolerable pain. Subjects in both groups were assured freedom to withdraw from testing at any time. If any medical complication occurred, it was appropriately investigated and treated by a physician.

\section{Test protocols}

All the subjects performed a group specific incremental cycling exercise test. The healthy subjects used a stationary friction loaded cycle ergometer (Monark Model 834, Made in Sweden) and the SCI subjects used an ERGYS II FES cycle ergometer (Therapeutic Technologies Inc., Alpha, OH, USA). In the healthy group, the test was initiated with a 2 min rest stage while the subject was seated on the cycle ergometer. During this period, baseline metabolic and NIRS measurements were recorded. Exercise then commenced with $2 \mathrm{~min}$ of free pedaling at $50 \mathrm{rpm}$, 
Table 1 Characteristics of subjects

\begin{tabular}{|c|c|c|c|c|c|c|}
\hline Subject & Gender & Age (years) & Weight (kgs) & Injury level & Injury duration (years) & FES training \\
\hline $\mathrm{H}-1$ & $\mathrm{~F}$ & 28 & 73 & $\mathrm{~N} / \mathrm{A}$ & $\mathrm{N} / \mathrm{A}$ & $\mathrm{N} / \mathrm{A}$ \\
\hline $\mathrm{H}-2$ & M & 33 & 85 & $\mathrm{~N} / \mathrm{A}$ & $\mathrm{N} / \mathrm{A}$ & $\mathrm{N} / \mathrm{A}$ \\
\hline $\mathrm{H}-3$ & M & 29 & 80 & $\mathrm{~N} / \mathrm{A}$ & $\mathrm{N} / \mathrm{A}$ & $\mathrm{N} / \mathrm{A}$ \\
\hline $\mathrm{H}-4$ & M & 42 & 91 & $\mathrm{~N} / \mathrm{A}$ & $\mathrm{N} / \mathrm{A}$ & $\mathrm{N} / \mathrm{A}$ \\
\hline $\mathrm{H}-5$ & $\mathrm{~F}$ & 48 & 70 & $\mathrm{~N} / \mathrm{A}$ & $\mathrm{N} / \mathrm{A}$ & $\mathrm{N} / \mathrm{A}$ \\
\hline H-6 & M & 48 & 80 & $\mathrm{~N} / \mathrm{A}$ & $\mathrm{N} / \mathrm{A}$ & $\mathrm{N} / \mathrm{A}$ \\
\hline $\mathrm{H}-7$ & M & 65 & 91 & $\mathrm{~N} / \mathrm{A}$ & $\mathrm{N} / \mathrm{A}$ & $\mathrm{N} / \mathrm{A}$ \\
\hline SCI-1 & $\mathrm{F}$ & 26 & 61 & T9 & 1 & None \\
\hline SCI-2 & M & 31 & 83 & $\mathrm{C} 5$ & 13 & Previous \\
\hline SCI-3 & M & 31 & 55 & $\mathrm{C} 5$ & 10 & Concurrent \\
\hline SCI-4 & M & 43 & 110 & $\mathrm{C} 5$ & 10 & Previous \\
\hline SCI-5 & $\mathrm{F}$ & 50 & 71 & $\mathrm{~T} 8$ & 18 & Concurrent \\
\hline SCI-6 & M & 50 & 74 & $\mathrm{~T} 4$ & 29 & Concurrent \\
\hline SCI-7 & M & 65 & 87 & $\mathrm{~T} 12$ & 3 & Concurrent \\
\hline
\end{tabular}

$\mathrm{H}=$ Healthy; SCI $=$ Spinal Cord Injured; N/A = Not Applicable

followed by 2 min stages in which the resistance was increased by $1 \mathrm{kp}$. The incremental exercise test was terminated when the maximum oxygen uptake $\left(\dot{\mathrm{V}} \mathrm{O}_{2 \max }\right)$ or voluntary fatigue was attained. The criteria ${ }^{22}$ for $\dot{\mathrm{VO}}_{2 \max }$ were attainment of two of the following three criteria: (a) leveling off or decline in the $\dot{\mathrm{VO}}_{2}$ with increasing work rate; (b) attainment of the age predicted maximum heart rate $(220-$ age, years), and (c) a respiratory exchange ratio $>1.10$. Upon completion of the test, the subjects were allowed to recover for $4 \mathrm{~min}$ in the following manner: $2 \mathrm{~min}$ of active recovery (pedaling without resistance) and $2 \mathrm{~min}$ of passive recovery (no pedaling) while remaining seated on the ergometer.

The SCI subjects performed cycle exercise on the ERGYS II FES cycle ergometer. Pedaling was induced by means of computer sequenced transcutaneous electrical stimulation of the leg extensor muscles (gluteus maximus and quadriceps) at a stimulation frequency of $50 \mathrm{~Hz}$ and current varying between 10 and $132 \mathrm{~mA}$. Electrodes coated with barrier gel were applied over each muscle belly. During FES cycling, a sensor continuously monitored pedaling cadence such that electrical stimulation produced a maximum of $50 \mathrm{rpm}$. As muscle fatigue occurred and cadence slowed, the intensity of the electrical stimulus was gradually increased to maintain the cadence at $50 \mathrm{rpm}$. In the SCI group, the maximum resistance a subject could attain was a multiple of $1 / 8 \mathrm{kp}$. In this study, five subjects could attain a maximum of $1 / 8 \mathrm{kp}$ whereas one subject could attain $2 / 8 \mathrm{kp}$ maximum and another $4 / 8 \mathrm{kp}$. The subjects followed a stage wise incremental protocol to voluntary exhaustion which was similar to that used for the healthy subjects. The first $2 \mathrm{~min}$ was a rest stage, which was followed by FES assisted cycling for $2 \mathrm{~min}$ with no resistance (ie free pedaling). The subject then cycled against a resistance starting at $25 \%$ of the individual maximal workload, increasing by an additional $25 \%$ every 2 min for four total stages. A minimum cadence of $50 \mathrm{rpm}$ was maintained during the test. When the pedaling cadence fell below $40 \mathrm{rpm}$ and electrical stimulation was maximal, the investigator provided some assistance to maintain the pedaling cadence at $50 \mathrm{rpm}$. This procedure is consistent with that previously described by Krauss et al. ${ }^{23}$ During recovery, the subjects performed $2 \mathrm{~min}$ of passive pedaling (investigator turning the pedals; no electrical stimulation) against no resistance followed by 2 min of resting recovery.

\section{Metabolic and cardiorespiratory measurements}

Metabolic and cardiorespiratory measurements were continuously monitored during the test using an automated metabolic measurement cart (MMC Horizon, Sensormedics Corporation, CA, USA). The metabolic cart was calibrated using commercially available precision gases $(16 \%$ oxygen, $4 \%$ carbon dioxide; $100 \%$ nitrogen). The calibration was verified at the end of the test to ensure accuracy of the data. The volume transducer was calibrated using a calibrated syringe. Respiratory gas exchange data were collected in the mixing chamber mode. The metabolic cart was programmed to present the following results every $15 \mathrm{~s}$ : absolute $\dot{\mathrm{VO}}_{2}(\mathrm{~L} / \mathrm{min})$, relative $\dot{\mathrm{VO}}_{2}(\mathrm{ml} / \mathrm{kg} / \mathrm{min}), \dot{\mathrm{V}}_{\mathrm{E}}(\mathrm{L} / \mathrm{min})$, and respiratory exchange ratio (RER). The HR was recorded during the last $10 \mathrm{~s}$ at each work stage using a wireless monitor (Sport Tester, Polar Pacer, Finland). The following variables were calculated from these data: (a) oxygen pulse $\left(\mathrm{O}_{2}\right.$ pulse, $\mathrm{ml} /$ beat $)$ as the ratio between absolute $\dot{\mathrm{VO}}_{2}$ and $\mathrm{HR}$, and (b) ventilatory equivalent for oxygen $\left(\dot{\mathrm{V}}_{\mathrm{E}} / \dot{\mathrm{VO}}_{2}\right.$ ratio) as the ratio between $\dot{\mathrm{V}}_{\mathrm{E}}$ and absolute $\dot{\mathrm{VO}}_{2}$. 
Near infrared spectroscopy measurements

NIRS measurements were continuously recorded during the rest, incremental exercise, and recovery stages using a commercial device (Runman, NIM Inc., PA, USA). The NIRS consists of three components: ${ }^{12}$ a probe with two tungsten lamps and two silicone diodes which record the tissue absorbency at 760 and $850 \mathrm{nM}$; an amplifier which magnifies the absorbency signal and displays it in millivolts; and a physiological recorder. In this study, the instrument was interfaced with an $\mathrm{A} / \mathrm{D}$ board to process and record the tissue absorbency signal at a frequency of $30 \mathrm{~Hz}$ as previously described. ${ }^{24}$ Prior to testing, the instrument was calibrated first at $760 \mathrm{nM}$ and then at $850 \mathrm{nM}$ as suggested by the manufacturer. Muscle oxygenation measurements were undertaken from the right vastus lateralis muscle according to procedures described by Belardinelli et al. ${ }^{25}$ The probe was placed on the skin over a motor point of the muscle, about $12-14 \mathrm{cms}$ from the anterior border of the knee, parallel to the major axis of the thigh. A piece of clear plastic was placed between the skin and the NIRS photo detectors, to prevent any possible distortion of the signal due to the accumulation of sweat on the diodes. A tensor bandage was used to secure the NIRS probe to the thigh without occluding blood flow to the muscle. Upon completion of the exercise and recovery phases of the test, the raw NIRS data were averaged over $15 \mathrm{~s}$ intervals so that these would correspond with the cardiorespiratory measurements obtained with the metabolic measurement cart. The values of tissue absorbency were plotted for the rest, incremental exercise and recovery stages to obtain a profile of the muscle oxygenation trend for each subject. The minimum value of tissue absorbency recorded during the incremental test was considered to be the maximum amount of deoxygenation in the muscle. ${ }^{11}$

\section{Statistical analysis}

Two way repeated measures analysis of variance (group by exercise stage) was used to make between group comparisons for the following variables of interest: absolute $\dot{\mathrm{V}} \mathrm{O}_{2}$, relative $\dot{\mathrm{V}} \mathrm{O}_{2}, \mathrm{HR}, \dot{\mathrm{V}}_{\mathrm{E}}, \mathrm{RER}$, $\mathrm{O}_{2}$ pulse, $\dot{\mathrm{V}}_{\mathrm{E}} / \dot{\mathrm{VO}}_{2}$ ratio and minimum tissue absorbency. For between and within group analysis, the first four exercise stages were analyzed since all the subjects (healthy and SCI) were able to attain this work stage. The Scheffe post hoc procedure was used to examine differences between the two groups. Multiple regression analysis was used to examine the relationship between $\mathrm{VO}_{2}$ and tissue absorbency during the transition from rest and the first four work stages and to compare the slopes of the regression equations between the healthy and SCI subjects. Values were considered to be significant at the 0.05 level of confidence. CSS Statistical computer programs were used to perform these analyses (Release 3.0, Tulsa, OK, USA).

\section{Results}

Physiological responses

The physiological and tissue oxygenation responses at rest and during peak exercise in the healthy and SCI subjects are summarized in Table 2. The trends for the $\dot{\mathrm{VO}}_{2}, \mathrm{HR}$ and $\dot{\mathrm{V}}_{\mathrm{E}}$ during rest, exercise and recovery for both groups of subjects are illustrated in Figures $1-3$ respectively. In the healthy subjects the $\dot{\mathrm{VO}}_{2}, \mathrm{HR}$ and $\dot{\mathrm{V}}_{\mathrm{E}}$ increased systematically from rest to maximal exercise. The $\dot{\mathrm{VO}}{ }_{2}$ and $\mathrm{HR}$ increased linearly with the work rate until the final stage of exercise, whereas the $\dot{\mathrm{V}}_{\mathrm{E}}$ increased in a curvilinear manner. None of these subjects reached the age predicted maximal HR (220-age, years), suggesting that the exercise test was terminated due to localized fatigue. However, they

Table 2 Physiological responses at rest and during peak exercise in the two groups

\begin{tabular}{lcccc}
\hline Variable & Rest & Healthy & & \multicolumn{2}{c}{ Spinal cord injured } \\
\hline $\begin{array}{l}\text { Oxygen uptake } \\
\text { L.min }\end{array}$ & $0.40 \pm 0.11$ & $3.08 \pm 0.95^{*}$ & $0.32 \pm 0.10$ & $0.65 \pm 0.18$ \\
$\begin{array}{l}\text { Oxygen uptake } \\
\text { ml.kg }^{-1} \text {.min }^{-1}\end{array}$ & $4.6 \pm 0.9$ & $38.2 \pm 9.3^{*}$ & $4.1 \pm 0.8$ & $8.3 \pm 2.1$ \\
$\begin{array}{l}\text { Ventilation rate } \\
\quad \text { L.min }\end{array}$ & $12.3 \pm 4.7$ & $130.4 \pm 22.7^{*}$ & $11.1 \pm 3.6$ & $31.6 \pm 14.4^{*}$ \\
$\begin{array}{l}\text { Heart rate } \\
\text { beats.min }\end{array}$ & $73 \pm 9.8$ & $175 \pm 6.7^{*}$ & $78 \pm 7.4$ & $83 \pm 8.2$ \\
$\begin{array}{l}\text { Respiritory exchange } \\
\text { ratio }\end{array}$ & $0.89 \pm 0.09$ & $1.33 \pm 0.09^{*}$ & $0.92 \pm 0.11$ & $1.24 \pm 0.12^{*}$ \\
$\begin{array}{l}\text { Oxygen pulse } \\
\text { ml.beat }\end{array}$ & $5.5 \pm 1.2$ & $17.5 \pm 5.6^{*}$ & $4.1 \pm 1.0$ & $7.8 \pm 2.4$ \\
$\begin{array}{l}\text { Ventilatory equivalent } \\
\text { for oxygen }\end{array}$ & $30.8 \pm 3.7$ & $42.1 \pm 6.1^{*}$ & $33.4 \pm 5.2$ & $47.5 \pm 8.4^{*}$ \\
$\begin{array}{l}\text { Tissue absorbancy } \\
\text { millivolts }\end{array}$ & $9 \pm 29$ & $-303 \pm 146^{*}$ & $-20 \pm 18$ & $-202 \pm 189^{*}$ \\
\hline
\end{tabular}

*Indicates significantly different from the resting value for the same group of subjects 
all exceeded the RER criterion of 1.10 suggesting a maximal effort on their part. In the SCI subjects, the pattern of the physiological responses during the incremental work stages differed from that observed

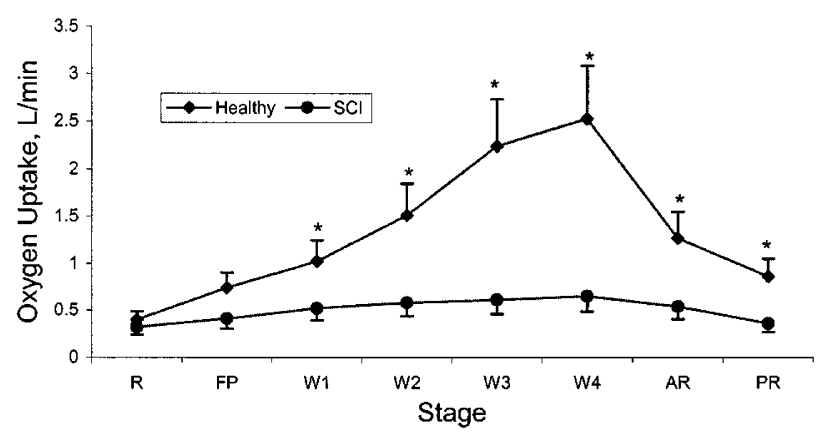

Figure 1 Oxygen uptake during the incremental work stages and recovery in the healthy and spinal cord injured subjects. The asterisk $(*)$ indicates a significant difference $(P<0.05)$ between the healthy and spinal cord injured subjects at that work stage. See text for details

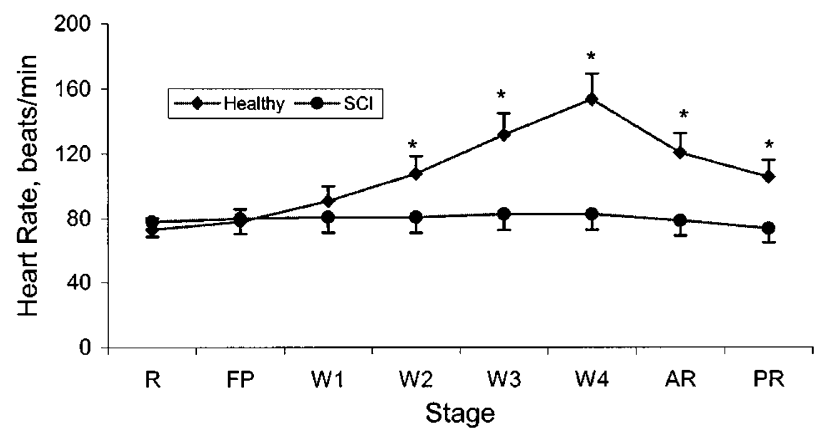

Figure 2 Heart rate during the incremental work stages and recovery in the healthy and spinal cord injured subjects. The asterisk $(*)$ indicates a significant difference $(P<0.05)$ between the healthy and spinal cord injured subjects at that work stage. See text for details

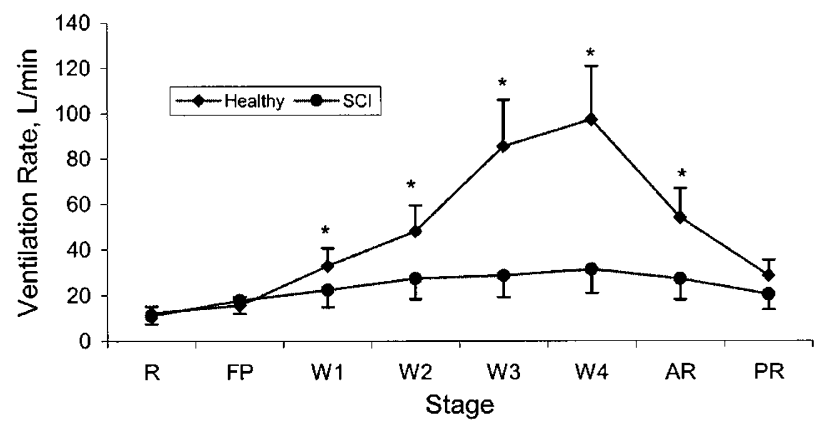

Figure 3 Ventilation rate during the incremental work stages and recovery in the healthy and spinal cord injured subjects. The asterisk $(*)$ indicates a significant difference $(P<0.05)$ between the healthy and spinal cord injured subjects at that work stage. See text for details in the healthy subjects. The $\dot{\mathrm{VO}}_{2}$ showed a small increase at each FES work stage and at the final stage the value was approximately twice the resting value. This difference, however, between the peak and resting value was not significantly different $(P>0.05)$ in these subjects. It is interesting to note that the increase in $\dot{\mathrm{VO}}_{2}$ in the SCI subjects was not accompanied by a linear increase in HR as was observed in the healthy subjects. A slight increase in HR was observed from rest to the final work stage (approximately 5 beats $/ \mathrm{min}$, $P>0.05)$ until the test was terminated due to fatigue. The $\dot{V}_{\mathrm{E}}$ increased significantly from rest with the peak values being approximately three times the resting values. With the exception of one SCI subject, all of them attained the criterion RER of 1.10 which is indicative of maximal exercise. Significant differences were observed between the two groups for each of these responses during exercise and recovery.

\section{Muscle oxygenation trends}

The trends in muscle oxygenation during the incremental work stages in the healthy and SCI subjects are illustrated in Figure 4. In the healthy subjects, there was an initial increase in tissue absorbency (ie increased oxygenation) at the onset of exercise (usually within the first few seconds), which was followed by a systematic decrease (ie decreased oxygenation) with increasing work rate. There was a tendency for the tissue oxygenation to level off as the maximum work rate was attained in some subjects (note: this leveling off is not illustrated in the figure because the data of seven subjects is averaged and only data for the four work stages is plotted). During active recovery, there was a very rapid increase in tissue absorbency towards the baseline value during the first $2 \mathrm{~min}$. This was followed by a further increase above the resting base line value during the remaining $2 \mathrm{~min}$ of passive recovery with a leveling off towards the end of this period. In the SCI subjects, the tissue absorbency trend differed from that observed in the healthy subjects. At the onset of FES exercise, the

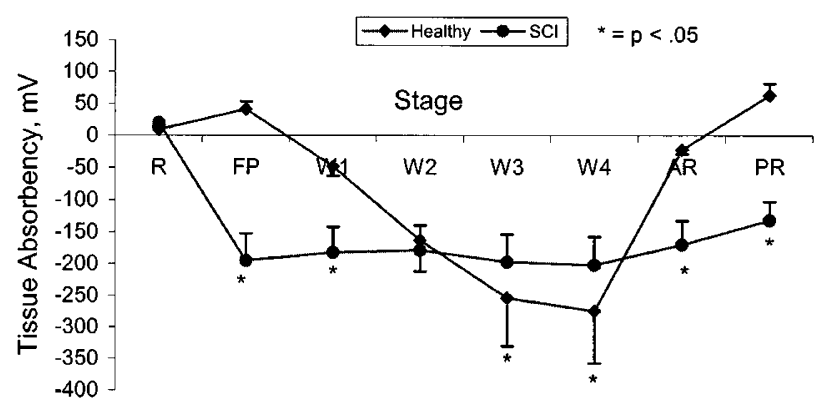

Figure 4 Tissue absorbency trends during the incremental work stages and recovery in the healthy and spinal cord injured subjects. The asterisk $(*)$ indicates a significant difference $(P<0.05)$ between the healthy and spinal cord injured subjects at that work stage. See text for details 
initial increase in tissue absorbency was not evident. Rather, there was an immediate decline in tissue absorbency of the vastus lateralis muscle that tended to remain stable for the remaining work stages. This trend was unlike the steady decline that was observed in the healthy subjects with increasing work rate. The minimum tissue absorbency observed in these subjects was significantly lower when compared to the healthy subjects. During the 2 min of active recovery, there was a modest increase in tissue absorbency towards the base line value. This was followed by a more gradual increase during the final $2 \mathrm{~min}$ of passive exercise. In the healthy subjects, the relative change in blood volume (determined by the sum of the 760 and $850 \mathrm{nM}$ signals) demonstrated a tendency for a small but systematic increase with increasing work rate, whereas in the SCI subjects this was not evident.

Results of the multiple regression analysis indicated that the common variance between the $\dot{\mathrm{VO}}_{2}$ and tissue absorbency during the transition from rest to the four work stages in the healthy subjects was best described by a linear model $\left(r^{2}=0.94\right)$, whereas in the SCI subjects the data were best represented by a curvilinear model $\left(r^{2}=0.66\right)$. However, in order to compare the rate of tissue absorbency between the two groups of subjects, the slopes and intercepts of the linear model were used for analysis. It is evident from the regression equations for the two groups presented in Figure 5 that the rate of decline in tissue absorbency per unit change in $\dot{\mathrm{VO}}_{2}$ was significantly faster in the SCI compared to the healthy subjects. The value of the intercept was not significantly different between the two groups (note: the difference in the slope of the two regression lines is not visualized because the scales for the $\mathrm{VO}_{2}$ on the $\mathrm{X}$ axis are different).

\section{Discussion}

Cardiorespiratory responses during incremental exercise The peak cardiorespiratory responses observed during FES cycling exercise to voluntary exhaustion observed in the SCI subjects are within the range of values previously reported. ${ }^{3,6,26}$ In the current study the $\mathrm{VO}_{2}$ increased by $100 \%(P>0.05)$ and was accompanied by a threefold increase in the $\dot{\mathrm{V}}_{\mathrm{E}}(P<0.05)$ and small increase in HR ( 5 beats $/ \mathrm{min}, P>0.05$ ) when compared to the resting values. Figoni et $a l^{3}$ and Hooker et $a l^{6}$ reported significant increases in each of these variables during FES exercise in SCI subjects. The most likely reason for the discrepancy between the present study and the ones cited is that the $\dot{\mathrm{VO}}_{2}$ and $\mathrm{HR}$ values recorded prior to the onset of exercise in this study were spuriously high. This could be attributed to the anticipation of exercise which is known to elevate the physiological responses under resting conditions. ${ }^{27} \mathrm{Had}$ these values been comparable to those reported in the studies cited, it is likely that these increases would also be significant. It should be pointed out that the subjects in the current study included three quad-

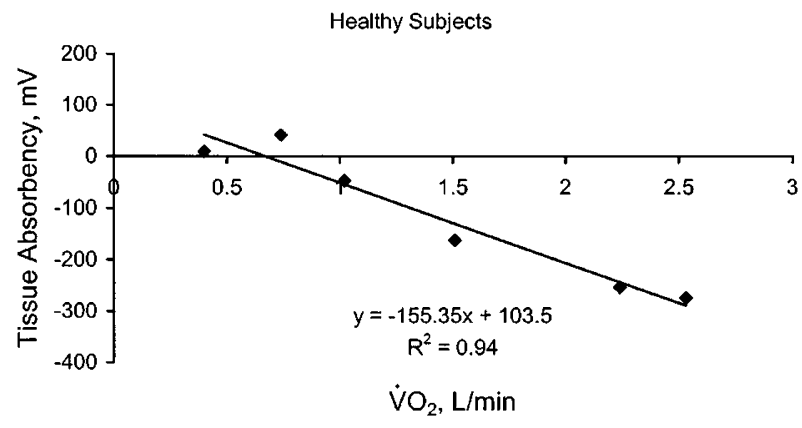

SCI Subjects

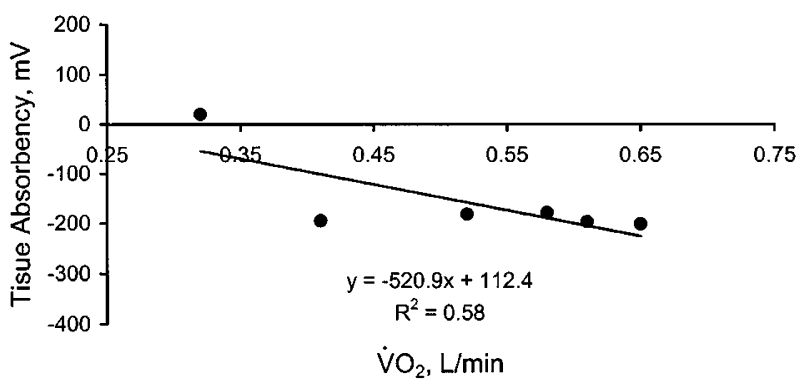

Figure 5 Regression between the absolute oxygen uptake and the tissue absorbency during the incremental work stages in healthy and spinal cord injured subjects. Note: The slope of the regression line was significantly steeper in the spinal cord injured subjects compared to that of the healthy subjects. This is not visible in the figure because of the smaller range of the oxygen uptake values on the $\mathrm{X}$ axis for the spinal cord injured subjects

riplegics and four paraplegics. Figoni et $a l^{3}$ compared the peak cardiorespiratory responses during FES cycle exercise between quadriplegics and paraplegics and reported similar trends in these responses in both groups. The paraplegics attained a higher power output than the quadriplegics $(15 \mathrm{~W}$ vs $9 \mathrm{~W})$ which resulted in values of $\dot{\mathrm{V} \mathrm{O}} 2, \mathrm{HR}$ and $\dot{\mathrm{V}}_{\mathrm{E}}$ that were higher by $19 \%$, $13 \%$ and $22 \%$ respectively. The authors attributed these differences in the peak hemodynamic responses to the autonomic integrity of the cardiorespiratory system rather than differences in levels of aerobic metabolism.

Hooker et $a l^{28}$ demonstrated a significant widening of the mixed $(\mathrm{a}-\mathrm{v}) \mathrm{O}_{2 \text { diff }}$ during submaximal FES cycle exercise in SCI subjects when compared to the resting value. This implies an increased oxygen extraction from the blood, which could be due to increases in aerobic metabolism of the muscles activated by FES and those indirectly (eg the heart and respiratory muscles) involved in exercise. Figoni et $a l^{3}$ compared the acute physiological responses during passive and active FES leg cycle exercise in subjects with SCI. They observed no significant increases in $\dot{\mathrm{VO}}_{2}, \mathrm{HR}$ and $\dot{\mathrm{V}}_{\mathrm{E}}$ above the resting levels during passive exercise, whereas the FES leg exercise elicited significant increases in each of these variables. The 
researchers concluded that the active muscular contractions induced by FES were essential for inducing the elevated metabolic and cardiorespiratory responses in these subjects.

It is likely that part of the increase in whole body $\dot{\mathrm{VO}}_{2}$ during the FES exercise was due to enhanced aerobic metabolism in the respiratory muscles, as evidenced by an increase in the $\dot{\mathrm{V}}_{\mathrm{E}}$ observed in the SCI subjects. Direct quantification of the $\mathrm{V}_{2}$ of the respiratory muscles during exercise is difficult. However, previous research indicates that the value increases exponentially with exercise intensity and ventilation rate. At rest and during light exercise, the $\dot{\mathrm{VO}}_{2}$ of the respiratory muscles is approximately $4 \%$ of the whole body $\dot{\mathrm{VO}}_{2}$, whereas during maximal exercise this value increases to $10 \%$ to $12 \%$, due to large increases in the depth and frequency of breathing. It also seems that the proportion of the $\mathrm{V}_{2}$ consumed by the respiratory muscles is significantly higher in the SCI subjects compared to healthy subjects. This is because the ventilatory equivalent for oxygen $\left(\dot{\mathrm{V}}_{\mathrm{E}} / \dot{\mathrm{VO}}_{2}\right.$ ratio), which is an index of the economics of ventilation, seems to be higher in the former group. The values of the $\dot{\mathrm{V}}_{\mathrm{E}} / \dot{\mathrm{VO}}_{2}$ ratio for the healthy vs SCI subjects during the transition from rest through the four work stages were: 32.5 vs 37.5, 17.6 vs 34.1, 20.0 vs 40.4, 20.1 vs 39.7 and 35.9 vs 4.0 respectively. It is interesting to note that the ratio was consistently higher in the SCI subjects compared to the healthy subjects. Muraki et $a l^{29}$ compared the cardiorespiratory responses during passive cycle exercise between healthy subjects and those with SCI. They reported that the $\dot{\mathrm{VO}}_{2}$ was significantly higher in the healthy subjects compared to the SCI subjects at pedaling cadences of 20 and $40 \mathrm{rpm}$. However, under each of these conditions, the $\dot{V}_{\mathrm{E}}$ was similar in the two exercise groups, implying that the $\dot{\mathrm{V}}_{\mathrm{E}} / \dot{\mathrm{V}} \mathrm{O}_{2}$ ratio was higher in the SCI group. It should be noted that the investigators cited $^{29}$ used passive cycle exercise by fixing the subject's limbs to the pedals of the cycle ergometer which were driven by a motor. Whether the physiological responses during this type of passive exercise differs from FES exercise is not known.

\section{Muscle oxygenation trends}

The muscle oxygenation trends during exercise and recovery observed in the healthy subjects were similar to those previously reported for this exercise mode. ${ }^{24,25}$ The increased oxygenation at the onset of exercise has been attributed to a redistribution of existing blood flow within the muscle ${ }^{25}$ and/or increased skin blood flow. ${ }^{30}$ The systematic decrease in oxygenation with increasing intensity implies a greater release of oxygen by hemoglobin/myoglobin via the Bohr effect. ${ }^{11,25}$ The leveling off in muscle oxygenation observed in some subjects at near maximal work rates suggests a peripheral limitation to exercise. Some important differences were observed in the muscle oxygenation trends between the healthy and SCI subjects. In the
SCI subjects, the initial increase in muscle oxygenation at the onset of exercise was not evident. Instead, there was an immediate decrease in muscle oxygenation during the free pedaling period, and thereafter, the level of muscle deoxygenation remained fairly stable throughout the remaining work stages. It is unclear whether the differences in tissue oxygenation at the onset of exercise between the two groups were due to differences in redistribution of intramuscular blood volume $^{25}$ and/or increased skin blood flow. ${ }^{30}$ The relative changes in blood volume, as indicated by the sum of the NIRS signal at the two wave lengths, showed a small but systematic increase in the healthy subjects but minimal change in the SCI subjects.

The fact that there was a decrease in muscle oxygenation during the initial work stages of FES exercise indirectly suggests that there was some active aerobic metabolism occurring in the paralyzed muscle of the SCI subjects. Further evidence that correlates the NIRS response with femoral blood oxygenation levels ${ }^{10,31}$ and alterations in cytochrome oxidase activity (an enzymatic marker for aerobic metabolism $)^{32}$ are needed to confirm these findings. It should be pointed out that the absolute amount of oxygen consumed by these muscles cannot be quantified by NIRS because the path length of the signal is not known. ${ }^{10,11}$ Difficulties associated with the scattering of photons once they penetrate the muscle tissue and the changing geometry of the muscle during exercise make this evaluation difficult.

The minimum tissue absorbency value observed in the SCI subjects was significantly lower than that observed in the healthy subjects. This suggests that the magnitude of muscle deoxygenation (ie the release of oxygen by hemoglobin and myoglobin) was lower in the SCI subjects compared to the healthy subjects. This could be attributed to the fact that the motor units of the paralyzed skeletal muscles of long-term SCI patients undergo a transformation from the Type I units that have a greater oxidative capacity to the Type II units which have a lower oxidative capacity. After the initial decline in tissue absorbency in the SCI subjects, the values remained fairly stable during the remaining work stages, implying that muscle deoxygenation had leveled off. It is likely, therefore, that the energy requirements during the latter stages of FES exercise were met by anaerobic metabolism. This is supported by the observations of Hooker $e t a l^{28}$ who reported that blood lactate accumulation during 30 min of submaximal FES exercise was unusually high for the power output and $\mathrm{VO}_{2}$ in persons with quadriplegia and paraplegia. It should be noted that accumulation of lactate has been implicated in muscle fatigue, ${ }^{33}$ and this may be contributing factor in the premature fatigue that is commonly observed in FES programs.

The current observations indicated that although the magnitude of muscle deoxygenation was lower in the SCI subjects, the rate at which the deoxygenation occurred per unit change in $\dot{\mathrm{VO}}_{2}$ was significantly 
faster in the SCI subjects. Barstow et al ${ }^{34}$ reported that the oxygen uptake kinetics (ie the time required to reach a submaximal steady state oxygen uptake) during FES exercise and recovery were significantly slower in subjects with SCI when compared to healthy, sedentary and ambulatory individuals. They attributed this to a reduced aerobic capacity of the stimulated muscles (preponderance of Type II motor units as indicated above) and/or an impaired cardiovascular response to the exercise stimulus. The fact that the tissue absorbency leveled off after the free pedaling period and during the four work stages support the findings of the researcher cited. The lack of the central cardiovascular command due to disruption of the sympathetic stimulation to the myocardium in the SCI subjects would reduce their overall oxygen transport capacity. ${ }^{35}$ The fact that the heart rate was significantly lower in the SCI subjects during the work stages supports this observation.

The results of the current study should be interpreted with caution for two reasons. Firstly, the maximum amount of deoxygenation of the vastus lateralis muscle induced by arterial occlusion was not determined for each subject. Recent studies ${ }^{31,36}$ have used a resting cuff ischemia protocol $(6-8 \mathrm{~min}$ of cuff ischemia using a blood pressure cuff inflated to $250 \mathrm{mmHg}$ ) to establish maximum tissue deoxygenation levels and have expressed the degree of deoxygenation during exercise relative to this level. This was not done in this study in light of the findings of Chance et al, ${ }^{11}$ who reported that the application of cuff ischemia to the vastus lateralis muscle following maximal exercise increased the maximum deoxygenation level by only $3 \%$. This suggests that the muscle was nearly maximally deoxygenated at the point of exhaustion. Secondly, the degree of deoxygenation could have been influenced by differences in the thickness of the skinfold layer between the two groups of subjects. It is likely that the SCI subjects had a thicker skinfold layer surrounding the vastus lateralis muscle ${ }^{8}$ due to significant atrophy of the skeletal muscle following the injury. Studies that have examined the influence of skinfold thickness on tissue deoxygenation using NIRS have indicated that the thickness of the fat layer ranging from 4 to $10 \mathrm{~mm}$ could result in a threefold difference in the tissue absorbency measurement. ${ }^{37,38}$ In this study, skinfold thickness at the NIRS measurement site was not recorded, and therefore, the values could not be corrected for this variable in the two groups of subjects. It should be noted, however, that the NIRS probe of this instrument is designed to have a penetration depth, which is approximately $60 \%$ of the distance between the light source and the optodes, or a penetration equivalent to $2.5-3 \mathrm{~cm}$. It appears that despite significant muscle atrophy and concomitant increase in skinfold fat in SCI subjects, this penetration depth seems to be sufficient to obtain proper NIRS recordings from the vastus lateralis muscle in this population. It is recommended that future studies comparing the tissue oxygenation status between SCI and healthy subjects: (a) utilize the cuff ischemia protocol to establish differences in the absolute degree of deoxygenation between these two groups, and (b) measure skinfold thicknesses at the NIRS measurement site to evaluate the influence of skinfold thickness on the tissue absorbency measurements.

In conclusion, the results of this study indicated that FES cycle exercise induced modest increases in the $\dot{\mathrm{V}} \mathrm{O}_{2}$ and $\dot{\mathrm{V}}_{\mathrm{E}}$ in subjects with SCI. These increases, however, did not result in a significant increase in the HR. Significant differences were observed between the healthy and SCI subjects for the deoxygenation trends during exercise and recovery. Although the SCI subjects demonstrated a smaller absolute change in muscle deoxygenation, the rate of change with respect to the change in absolute $\dot{\mathrm{VO}}_{2}$ was significantly faster in the SCI subjects. This was most likely due to the greater recruitment of the Type II motor units which are predominant in SCI subjects following injury. During recovery from exercise, muscle reoxygenation appeared to be delayed in the SCI subjects when compared to the healthy subjects. These observations support previous findings in oxygen uptake kinetics between these two groups of subjects.

\section{Acknowledgements}

The authors extend their appreciation to Dr Gordon Bell for his time and assistance towards this project.

\section{References}

1 Sykes L et al. Energy expenditure of walking for adult patients with spinal cord lesions using the reciprocating gait orthosis and functional electrical stimulation. Spinal Cord 1996; 34: 659-665.

2 Laskin JJ et al. Electrical stimulation-assisted rowing exercise in spinal cord injured people. Paraplegia 1993; 31: 534-541.

3 Figoni SF et al. Physiologic responses of paraplegics and quadriplegics to passive and active leg cycle ergometry. $J \mathrm{Am}$ Paraplegia Soc 1990; 13: 33-39.

4 Davis GM et al. Cardiovascular responses to arm cranking and FNS-induced leg exercise in paraplegics. J App Physiol 1990; 69: $671-677$.

5 Nash MS et al. Effects of electrically stimulated and passive motion on echocardiographically-derived wall motion and cardiodynamic function in tetraplegic persons. Paraplegia 1995; 33: $80-89$.

6 Hooker SP et al. Metabolic and hemodynamic responses to concurrent voluntary arm crank and electrical stimulation leg cycle exercise in quadriplegics. J Rehabil Res Dev 1992; 29: 1 - 11 .

7 Burnham $\mathrm{R}$ et al. Skeletal muscle fibre type transformation following spinal cord injury. Spinal Cord 1997; 35: 86-91.

8 Mohr T et al. Long term adaptation to electrically induced cycle training in severe spinal cord injured individuals. Spinal Cord 1997; 35: $1-16$.

9 Martin TP, Stein RB, Hoeppner PH, Reid DC. Influence of electrical stimulation on the morphological and metabolic properties of paralyzed muscle. J Appl Physiol 1992; 72: $1393-$ 1400.

10 Mancini D et al. Validation of near-infrared spectroscopy in humans. J Appl Physiol 1994; 77: 2740-2747. 
11 Chance B et al. Recovery from exercise-induced saturation in the quadriceps muscles of elite competitive rowers. Am J Physiol 1992; 262: C766-C775.

12 Wilson $\mathrm{J}$ et al. Noninvasive detection of skeletal muscle underperfusion with nearinfrared spectroscopy in patients with heart failure. Circulation 1989; 80: $1668-1674$

13 Simonson SG, Piantidosi CA. Near-infrared spectroscopy: clinical applications. Critical Care Clin 1996; 12: 1019-1029.

14 Belardinelli R, Georgiou D, Barstow TJ. Near infrared spectroscopy and changes in skeletal muscle oxygenation during incremental exercise in chronic heart failure: a comparison with healthy subjects. G Ital Cardiol 1995; 25: 715-724.

15 Matsui $\mathrm{S}$ et al. Assessment of working skeletal muscle oxygenation in patients with chronic heart failure. Am Heart $J$ 1995; 129: $690-695$.

16 Mancini $\mathrm{D}$ et al. Respiratory muscle deoxygenation during exercise in patients with heart failure demonstrated with nearinfrared spectroscopy. J Am Coll Cardiol 1991; 18: 492 - 498.

17 McCully KK, Halber C, Posner JD. Exercise-induced changes in oxygen saturation in the calf muscles of elderly subjects with peripheral vascular disease. J Gerontol Biol Sci 1994; 49: B128B134.

18 Brett GA et al. Near-infrared spectroscopy for monitoring of tissue oxygenation of exercising skeletal muscle in a chronic compartment syndrome model. J Bone Joint Surg 1997; 79A: $838-843$

19 Mohler $\mathrm{R}$ et al. Intramuscular deoxygenation during exercise in patients who have chronic anterior compartment syndrome of the leg. J Bone Joint Surg 1997; 79A: 844-849.

20 Physical Activity Readiness Questionnaire. Canadian Society for Exercise Physiology, Gloucester, Ontario, 1994.

21 Ashley EA et al. Evidence of autonomic dysreflexia during functional electrical stimulation in individuals with spinal cord injuries. Paraplegia 1993; 225: $593-605$.

22 American College of Sports Medicine. Guidelines for Exercise Testing and Prescription. Lea \& Febiger, Philadelphia, 1991.

23 Krauss JC et al. Effects of electrical stimulation and upper body training after spinal cord injury. Med Sci Sports Exer 1993; 25: $1054-1061$

24 Bhambhani Y, Buckley S, Susaki T. Detection of ventilatory threshold using near infrared spectrometry in men and women. Med Sci Sports Exer 1997; 29: 402 - 409.
25 Belardinelli R, Barstow T, Porsasz J, Wasserman K. Changes in skeletal muscle oxygenation during incremental exercise measured with near infrared spectroscopy. Eur J Appl Physiol 1995; 70: $487-492$

$26 \mathrm{Kjaer} \mathrm{M}$ et al. Heart rate during exercise with leg vascular occlusion in spinal cord-injured humans. J Appl Physiol 1999; 86: $806-811$.

27 Astrand PO, Rodahl K. Textbook of Work Physiology Physiological Bases of Exercise. McGraw-Hill Book Co., New York, 1986.

28 Hooker SP et al. Physiologic responses to prolonged electrically stimulated leg-cycle exercise in the spinal cord injured. Arch Phys Med Rehabil 1990; 71: $863-869$.

29 Muraki S et al. Cardiovascular and respiratory responses to passive leg cycle exercise in people with spinal cord injuries. Eur $J$ Appl Physiol 1996; 74: 23-28.

30 Maehara $\mathrm{K}$ et al. Effect of hypoxia and carbon monoxide on muscle oxygenation during exercise. Am J Resp Crit Care Med 1997; 155: $229-235$.

31 MacDonald MJ, Tarnopolsky MA, Green HJ, Hughson RL. Comparison of femoral blood gases and muscle near-infrared spectroscopy at exercise onset in humans. J Appl Physiol 1999; 86: $687-693$

32 Hampson N, Piantadosi C. Near infrared monitoring of human skeletal muscle oxygenation during forearm ischemia. $J \mathrm{Appl}$ Physiol 1988; 64: 9-57.

33 Roberts D, Smith DJ. Biochemical aspects of peripheral muscle fatigue. Sports Med 1989; 9: 125-138.

34 Barstow TJ et al. Gas exchange kinetics during functional electrical stimulation in subjects with spinal cord injury. Med Sci Sports Exer 1995; 27: $1284-1291$.

35 Mitchell JH, Victor RG. Neural control of the cardiovascular system: insights from muscle sympathetic nerve recordings in humans. Med Sci Sports Exer 1996; 28: S60-S69.

36 Foster $\mathrm{C}$ et al. Evidence for restricted muscle blood flow during speed skating. Med Sci Sports Exer 1999; 31: 1433-1440.

37 Homma S, Fukunaga T, Kagaya A. Influence of adipose tissue thickness on near infrared spectroscopic signal in the measurement of human muscle. J Biomedical Optics 1996; 1: 418-424.

38 Yamamoto $\mathrm{K}$ et al. Accurate NIRS measurement of muscle oxygenation by correcting the influence of a subcutaneous layer. SPIE 1998; 3194: $166-173$. 